\title{
ESTUDO DA TÉCNICA DE COLETA, CONGELAÇÃO E DESCONGELAÇÃO DE EMBRIÕES DE CAPRINOS (Capra hircus), DA RAÇA SAANEN, PORTADORES DA TRANSL,OCAÇÃO 5/15
}

\author{
STUDY OF EMIBRYOS COLECT TECHNIQUE, IROZEN AND THAWING OF SAANEN \\ (Capra hircus), GOATS BEARING 5/I5 TRANSLOCATION
}

Nereu Carlos PRESTES'; Cezinande de MFIRA²; Carlos Antonio de Miranda BOYfIM³; Sony IDimas HICUDO'; Maria Denise LOPES'; Frederico ()zanam PAPA ${ }^{+}$

\begin{abstract}
RESUMO
No presente trabalho foi realizado um estudo da técnica cirúrgica de coleta de embriòes na espécie caprina. utilizando-se doadoras heterozigotas para a translocação 5/15. acasaladas com animal translocado. Simultaneamente foram observados aspectos técnicos inerentes à crioprescrvação, descongelação e cultivo das estruturas recuperadas. Frente ao tratamento superovulatório, o cio foi observado em 24 ou 48 horas após a remoção da esponja intravaginal e foram recuperadas 71 estruturas viáveis $\mathrm{cm} 43$ coletas. O I-2 Propanodiol mostrou-se eficiente criopreservador, permitindo cultivo de todos os embriōes, fato não observado quando utilizouse o Gilicerol. As aderências provocadas pelas repetidas coletas constituem-se no grande desafio no sentido de se aperfeiçoar a técnica. pois são consideradas um fator limitante ao uso de doadores no início da vida reprodutiva.
\end{abstract}

UNITERMOS: Embriões; Criopreservaçāo; Descongelação; Caprinos

\section{INTROIDUÇÃO E LITERATURA}

Historicamente, o desenvolvimento das técnicas de coleta, congelação, descongelação e transferência de embriões tem duas fases.

O primeiro trabalho utilizando a cabra como material experimental no estudo da morfologia embrionária foi realizado por AMOROSO et al.' (1942). Em 1963, NISHIKAWA c ONUMA". verificaram guc o ovário de fêmeas da raça Saanen loi extremamente sensivel à ação do PSH como indutor de superovulação.

MOORE (1974) utilizou esponjas intravaginais de poliuretano, embebidas com progesterona com o intuito de induzir o cio em caprinos, permanecendo inseridas por 16 a 22 dias, obtendo excelentes resultados. O mesmo autor coletou embriões de cabras doadoras, 96 a 132 horas após a primeira cobertura, por meis cirúrgico, pela linha branca, sob anestesia geral (Nembutal). Avaliou a reação ovariana, lavou tubas c cornos uterinos para recuperar embriōes, empregando PBS (Phosphate Buffered Saline) como meio, enriquecido com $10 \%$ de soro de cabra heterólogo. Referiu média superior a 7 embriões por doadora.

NUTI ct al. ${ }^{10}$ (1987) trabalhando com 35 cabras pura. Nubianas e Alpinas, promoveram coleta cirúrgica de embriões aos 7 dias após a cobertura. Foram recuperadas 36 estruturas ( $X=$ 1,02), lavando a tuba e cornos uterinos pela inserção de catéter tipo Foley número 8 e $50 \mathrm{ml}$ de PBS, pH 7,2 com $5 \%$ de soro de albumina bovina e $1 \%$ de antibiótico.

BARII, et al. ${ }^{2}$ (1989) lavaram tubas e cornos uterinos de caprinos superovulados recolhendo $17 \mathrm{embriões} \mathrm{de} 6$ dias das 52 doadoras operadas.

BILTON e MOORE (1976) trabalhando com cultivo $\mathfrak{c}$ preservaçăo de embriōes caprinos congelaram 7 estruturas cm palheta contendo $0.3 \mathrm{ml}$ de $\mathrm{PBS}+25 \%$ de soro de cabra hetcrólogo, adicionado de $2 \mathrm{M}$ de glicerol ou (0,4 $\mathrm{M}$ de I)MSO. Reduziram a temperatura $1,0^{\circ} \mathrm{C} /$ minuto, até $6^{\circ} \mathrm{C}$, permanecendo por 40 minutos. Posteriormente as palhetas foram transferidas para recipiente contendo etanol gelado, reduzindo a temperatura em 0 a 2 ou 1 a $4^{\circ} \mathrm{C} /$ minuto. A cristalização foi induzida aos $-2,5^{\circ} \mathrm{C}$. Alingindo $-60^{\circ} \mathrm{C}$ as palhetas foram mergulhadas em nitrogênio líquido. A descongelação foi promovida em etapas de $8^{\circ} \mathrm{C} /$ minuto. Sugeriram que o glicerol foi mais efetivo como crioprotetor que o DMSO.

Scgundo POLGE e WIL I ADSEN" (1978), estruturas embrionárias em idades diferentes e de espécies distintas apresentaram grau de tolerância variável à congelação.

\footnotetext{
- Professor Assistente Doutor - Faculdade de Meclicina Veterinária e Zentecnia da UNESP - Campus de Butucutu

Professor Assistente - Faculdade de Medicira Veterinária e Zootecnín da LNFEP Campus de Botucatu

Médico Veterinário - Residente - Faculdade de Medicina Vetennária e Zootecnia da UNESP - Campus de Botucatu

- Professor Adjunto - Faculdade de Medicina Veterinária e Zootecnia da UNESP - Campus de Hotucatu
} 


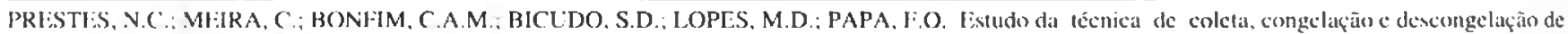

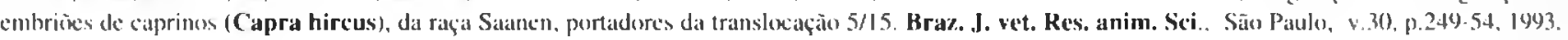
Suplemento.

Para BO()"'RON'(1984), uma criteriosa avaliação da cristalização deve ser eletuada, pois este foi o ponto crítico dos danos provocados à célula durante a congelação. Notou que a máxima cristalização do gelo foi menos intensa com o l-2 Propanodiol do que com o glicerol.

Para HALASZ e COLLINS" ( 1984$)$; WILDT el al. ${ }^{13}$ (1986); FRIELDLER el al. (1988), o Propanodiol pode ser menos tóxico que o glicerol e o DMSO. Concordaram também que dada a sua estabilidade a baixas temperaturas, o Propanodiol limitou a formação de cristais de gelo durante a congelaçāo e descongelação. Admitiram ainda que pode ser utilizado com outros agentes na tentativa de reduzir os danos osmóticos e a toxicidade específica.

Segundo FRIELDLER et al." (1988) a sucrose não penetra na célula e scu cfeito protetor foi verificado submetendo células a diluições sucessivas após a descongclação rápida como intuito de reidratação.

BARIL et al. (1989) promoveram a descongelação de 203 embriōes de caprinos, mergulhando as palhetas em água a $37^{\circ} \mathrm{C}$ por 30 segundos, obtendo índice de sucesso de $68 \%$.

O objetivo deste trabalho foi testar métodos de indução do cio e de superovulação, bem como promover a coleta cirúrgica, congelação e descongelação de embriōes em um grupo de cabras da raça Saanen sabidamente portadoras de translocação $5 / 15$.

\section{MATERIAL E MÉTODO}

\section{Animais}

Foram utilizadas 16 cabras adultas da raça Saanen, com sanidade física e fertilidade comprovadas, portadoras de translocação $5 / 15 \mathrm{~cm}$ estado heterozigoto, cruzadas com macho Saanen translocado heterozigoto. No mesmo grupo de animais, procederam-se 21 tentativas de colcta na cstação de atividade reprodutiva de 1989 e 22 intervençōes na estação cíclica de 1990.

\section{Indução de Cius c Método de Superovulação}

Aplicaram-se três fórmulas baseadas em métodos propostos

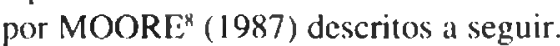

Cada esponja vaginal de espuma sintética, recebeu, 24 horas antes do uso, $1 \mathrm{ml}$ de Penicilina e $60 \mathrm{mg}$ de Acetato de Medroxiprogesteronab, deixadas secar à temperatura ambientc.

a = Pentabiótico Veterinánio - 1.200.000 UI FONTOLRA WYETH S/A $\mathrm{b}=$ PROMONE - E - $50 \mathrm{mg} / \mathrm{ml}$ - Solução aquosa estéril UPJOHN S/A $c=$ CIOSIN - Prostaglandina Sintérica (cloprostenol) COOPERS DO BRASIL, S/A $\mathrm{d}=$ FSH-P (Follicle Stimulating Hormone - Pituitary) $50 \mathrm{mg}$ - SHERING CORPORATION USA
O cio foi desencadeado pela aplicação do Cloprostenol' e a superovulação toi estimulada pelo uso de FSH-p.l.

\section{Programa I}

Iniciado I 0 dias após a observação do cio natural, ou induzido pelo uso de esponja intravaginal.

\begin{tabular}{|c|c|c|}
\hline D)10 - & tarde & $-4 \mathrm{mg} \mathrm{FSH}^{\prime \prime}$ \\
\hline D11 - & $\begin{array}{l}\text { manhã } \\
\text { larde }\end{array}$ & $\begin{array}{l}-2 \mathrm{mg} \mathrm{FSH} \\
-\quad 2 \mathrm{mg} \mathrm{FSH}\end{array}$ \\
\hline DI2 - & $\begin{array}{l}\text { manhã } \\
\text { tarde }\end{array}$ & $\begin{array}{l}-2 \mathrm{mg} \text { FSH } \\
-2 \mathrm{mg} \text { FSH }+100 \text { ug Cloprostenol* }\end{array}$ \\
\hline D13 - & $\begin{array}{l}\text { manhã } \\
\text { tarde }\end{array}$ & $\begin{array}{l}-2 \mathrm{mg} \text { FSH }+100 \mathrm{ug}(0.4 \mathrm{ml}) \\
\text { Cloprostenol } \\
-\quad 2 \mathrm{mg} \mathrm{liSH}\end{array}$ \\
\hline
\end{tabular}

DI4 ou D15

- Obscrvação de cio e cobertura

\section{Programa II}

DZero - Inserção da csponja intravaginal

$\begin{array}{lll}\text { D8 - } & \begin{array}{l}\text { manhã } \\ \text { tarde }\end{array} & -4 \mathrm{mg} \mathrm{FSH} H^{\lrcorner} \text {c } 100 \text { ug Cloprostenol } \\ \text { D9 - } & \begin{array}{l}\text { manhã } \\ \text { tarde }\end{array} & -2 \mathrm{mg} \text { FSH } \\ & -2 \mathrm{mg} \mathrm{FSH} \\ \text { DI0 - } & \begin{array}{l}\text { manhã } \\ \text { tarde }\end{array} & -2 \mathrm{mg} \text { FSH } \\ \end{array}$

D11 ou D12 - Cioc cobertura

\section{Programa III}

D\%ero Colocação da esponja intravaginal

$\begin{array}{lll}\text { D10 - manhã } & -4 \mathrm{mg} \text { FSH } \\ \text { tarde } & -4 \mathrm{mg} \text { FSH } \\ \text { D11 - } & \text { manhã } & -3 \mathrm{mg} \text { FSH } \\ \text { tarde } & -3 \mathrm{mg} \text { FSH } \\ \text { D12 - } & \text { manhã } & -2 \mathrm{mg} \text { FSH } \\ & \text { tarde } & -2 \mathrm{mg} \text { FSH c } 150 \text { ug Cloprostenol } \\ & & \text { Remoção da esponja }\end{array}$

D13 - D14 ou D15 - Cio e cobertura 


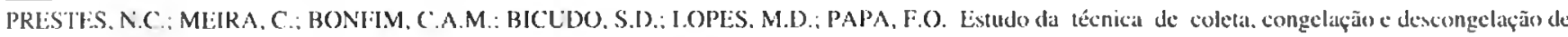

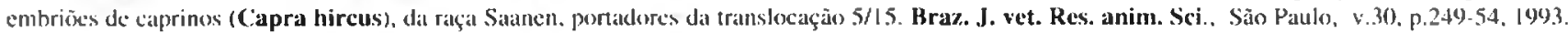
Suplemento.

Para deteção do cio foi utilizado macho vasectomizado como rufião, colocado em contacto com as fêmeas, em intervalos de 12 horas.

\section{Coleta de Embriões}

A coleta foi efeluada 5 a 6 dias após a cobertura. Os animais foram submetidos a hipnoanalgesia utilizando Cloridrato de Xilazina e infiltração local com Xilocaaína a $2 \%$. Seguiu-se realizando a incisão de aproximadamente $15 \mathrm{~cm}$ de comprimento, preferencialmente no flanco esquerdo, expondo o trato reprodutivo. Os ovários foram observados a fim de estimar o sucesso da coleta. A tuba foi lavada inserindo-sc tubo plástico de diâmetro compatível, adaptado em agulha, guiado e interiorizado através da fímbria, injetando-se lenta e suavemente 3 a $5 \mathrm{ml}$ de solução de $\mathrm{PBS}^{2}$ aquecida a $37^{\circ} \mathrm{C}$. fluindo em direção ao corno uterino.

A lavagem do útero foi efetuada a seguir, através de pequena incisão ( 1 a $1.5 \mathrm{~cm}$ ), próxima à hifurcação. Foi introduzido, em direção ao corno uterino, um catéter de Foley pediátrico $n^{\circ} 9\left(25 \mathrm{~cm}\right.$ - bulbo $\left.3,0 \mathrm{~cm}^{3}\right)$, ou sonda endotraqueal' infantil, $n^{\circ} 3$ a 3.5 , cujo balonete foi inflado com ar, prevenindo o refluxo do meio. Na extremidade oposta, próximo à junção útero-tubárica foi inserida uma agulha $50 \times 15$ ou catéter de Teflon' (14x,5,5). O corno uterino foi lavado pelo lluxo com turbilhonamento do PBS a $37^{\circ} \mathrm{C}, 20$ a $40 \mathrm{ml}$, em ambos os sentidos, recolhendo o meio em placa de Petri ${ }^{h}$ estéril aquecida. Repetiu-se a operação no corno contralateral.

A incisão aberta no útero foi suturada em modelo invaginante utilizando-se categute 3-() agulhadol. A síntese da parede abdominal foi realizada em dois planos de sutura contínua com fïo absorvível número $2^{n 1}$ e a sutura da pele foi efetuada em pontos isolados (L'horizontal), com fio de algodãon.

Omeio recuperado foi examinadosob lupa". a lim de quantificar e qualificar os embriões.

\section{Preparo - Congelação}

Para o Grupo I (Tab. 3), a desidratação foi processada por passagens sucessivas das estruturas durante 8 minutos cada. conforme o escuema abaixo:

\section{Solução 1}

$4.5 \mathrm{ml} \mathrm{PBS}+0.5 \mathrm{ml} \mathrm{SF} \mathrm{B}^{\prime}(10 \%)+0.18 \mathrm{ml}$ Glicerol $(3,3 \%)$

$c=$ Runpum - BAYIER DO BRASII. S/A

$\mathrm{f}=$ Cloridrato de Lindocaína - LEBPJ:TIT S/A

$\mathrm{g}=$ Dulbeceo madificado $.500 \mathrm{ml} \quad$ CLII.TIL.AB

h = Latex Foley C'atheter - com válvula - PI:RRY - USA

$i=$ Rusch 14.16, W. Germany $W_{\text {in }} n^{\circ} 3$ su Bn n* 3.5

$\mathrm{i}=$ Radiupaque fep Tetlon I. V. Catheter T $1+$ - (ixS 1/2 - ABBOT - USA

\section{Solução 2}

$4,5 \mathrm{ml} \mathrm{PBS}+0,5 \mathrm{ml} \mathrm{SFB}(10 \%)+0,36 \mathrm{ml}$ Glicerol $(6,6 \%)$

\section{Solução 3}

$4,5 \mathrm{ml} \mathrm{PBS}+0.5 \mathrm{ml} \mathrm{SFB}(10 \%)+0.36 \mathrm{ml} \mathrm{Glicerol}(10.0 \%)$

Para o grupo 2 (Tab. 3) a desidratação foi cfetuada por passagens sucessivas das estruturas, conforme segue:

\section{Solução 1}

PBS $+20 \%$ SF $B+0,75 \mathrm{M}$ de Propanodiol durante 5 minutos

\section{Solução 2}

PBS + 20\% SFB + 1.50 M de Propanodiol durante 10 minutos

Após este tempo, os embriões foram envasados em palhetas" de $0,5 \mathrm{ml}$. previamente identificadas e congelados pelo método rápido, utilizando o cilindro de Peter Elsden adaptado na boca do botijão de nitrogênio líquido (SOU/.A; DE BEM ${ }^{12}$, 1988).

A curva de congelamento utilizada, obedeceu () seguinte gráfico (Fig. 1).

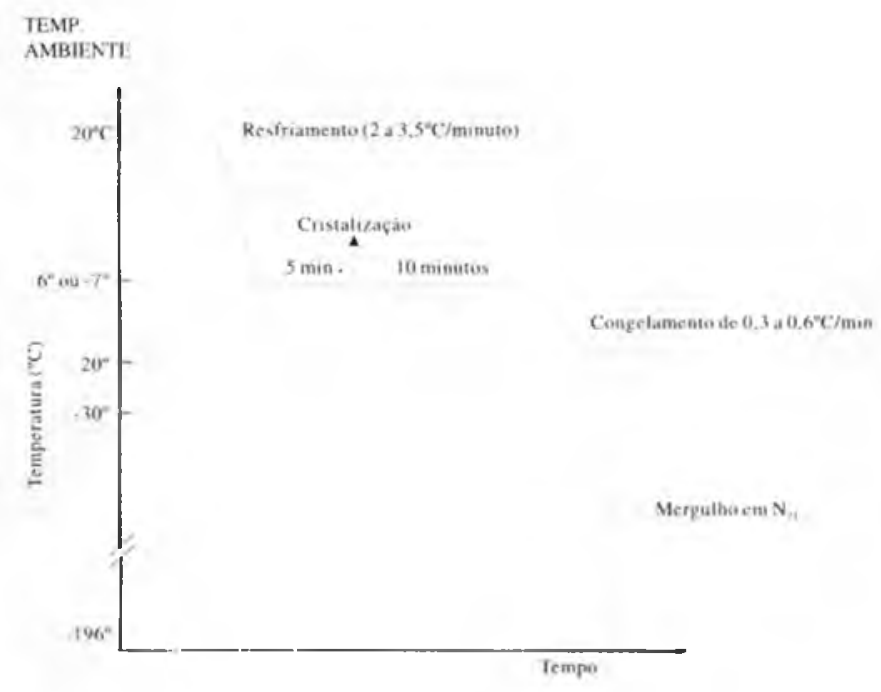

FIGLiRA 1

Gráfico da Curva de Resfriamento e Congelação de Embriões Caprinos realizada com cilindro na Boca do Botijão. Botucatu - SP, 1991

\footnotetext{
$k=$ Placas de Pelry PYRI:X - 160)X15

1 = Calcgute cromado 3-0 - 75vm-BRASMRIDICA S/A

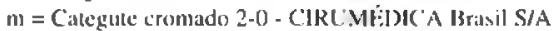

$\mathrm{n}=$ Linha Mercerizadil - (M) - CORRFNTI: S/A I,TI)

o = Estercomicroscópio CARL ZEISS - JENA MICRONAL - MICRONAI

$\Gamma=S H B$ soro fetal bovino

$y=$ Pallhetas de polietilenu-PAILLETLS BOVINES - RIEF A.A 101-I.M.V
} 


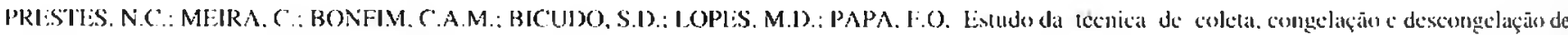

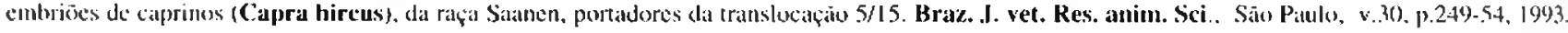
Suplemento.

\section{Descongelamento e Reidratação}

Parao Grupo I as palhetas foram mergulhadas em água a $37^{\circ} \mathrm{C}$ por 20 segundos e as estruturas foram submetidas a três passagens sucessivas, conforme segue:

\section{I -4.5 mI HAM S FI0 + 10\% SFB + Glicerol $6.6 \%-8$ minutos;}

$2-4,5 \mathrm{ml} \mathrm{H} A \mathrm{M}^{\top} \mathrm{S} F \mathrm{FI}+10 \% \mathrm{SFB}+$ Glicerol $3,3 \%-8$ minutos;

$3-4.5 \mathrm{ml} \mathrm{IIAM} \mathrm{S}$ F $10+10 \%$ SI'B -8 minutos.

Parao (irupo 2 as palhetas congeladas foram mergulhadas em água a $37^{\circ} \mathrm{C}$ por 20 segundos e as estruturas foram submetidas a quatro passagens sucessivas em solução de Propanodiol c Sucrose.

I - 1,0 M Propanodiol + 0,2 M Sucrose - 6 a 7 minutos;

2 - 0.5 M Propanodiol + 0,2 M Sucrose - 6 a 7 minutos;

3 - 0,0 M Propanodiol + 0,2 M Sucrose - 6 a 7 minutos;

4 - PBS puro su HAM'S FI0.

\section{RESULTADOS}

\section{TABELA}

Tempo de ocorrência do cio após a remoção da esponja intravaginal nos animais cujo estro foi induzido. Botucatu SP. 1991.

\begin{tabular}{|c|c|c|c|c|c|c|}
\hline \multirow{2}{*}{ ANO } & \multirow{2}{*}{$\begin{array}{l}\text { N"IDE: } \\
\text { ANIMAIS }\end{array}$} & \multirow{2}{*}{$\begin{array}{c}\text { ('IC) NAO } \\
\text { OBSERVADO }\end{array}$} & \multicolumn{4}{|c|}{ NL'MIIRO DE ANIMAIS EMCIO } \\
\hline & & & $12 \mathrm{~h}$ & $2+h$ & 3hin & t8h \\
\hline 1989 & 21 & 2 & 0 & 8 & 1 & 10 \\
\hline |ख⿻1 & 22 & 1 & 0 & 10 & 0 & 11 \\
\hline TSTAS & +3 & 3 & 0 & 18 & 1 & 21 \\
\hline
\end{tabular}

Em todos os animais, no ato da remoção da esponja, foi observado corrimento vaginal, diagnosticado como vaginite por reação a corpo estranho, que recuperava em 2 a 3 dias sem tratamento.

TABEILA 2

Número de estruturas colhidas na dependência do tratamento superovulatório imposto. promovendo a lavagem das tubas e cornos uterinos. Botucalu - SP, 1991.

\begin{tabular}{|c|c|c|c|}
\hline PROGRAMA & $\begin{array}{c}\text { NIMI:ROJBI: } \\
\text { ANIMAIS } \\
\text { TRATAIXS }\end{array}$ & $\begin{array}{l}\text { ES]RLTURAS } \\
\text { VIAVIISS }\end{array}$ & $\begin{array}{l}\text { ISTIRITURARS } \\
\text { INVIAVIIS }\end{array}$ \\
\hline II & 27 & 58 & 3 \\
\hline III & 1 & 4 & () \\
\hline 1 & 15 & c) & 0 \\
\hline TOTAL & 4.3 & 71 & 3 \\
\hline
\end{tabular}

\section{TABELA 3}

Número de estruluras congeladas com diferentes criopreservadores e embriōes cultivados a fresco. Botucatu SP, 1991 .

\begin{tabular}{|c|c|c|c|}
\hline GRI:PO & $\begin{array}{l}\text { NLGIERUDE } \\
\text { I:STRUTL RAS }\end{array}$ & CRIOPRFSERVAXOR & $\begin{array}{l}\text { ('RI:SCIMIVTO } \\
\text { I:U ("ULTIVO* } \\
\text { (VIABILIDADË) }\end{array}$ \\
\hline 1 & .88 & Glicerol & \\
\hline 2 & 20 & 1-2 Prapanoxdisl & + \\
\hline 3 & 13 & Atreser & + \\
\hline TOTAL & 71 & & \\
\hline
\end{tabular}

* Meio de cultura: PI3S + Soro fetal bovino loc ou HAM'S F10 (CULTILAB).

\section{TABELA 4}

Resposta ovariana ao tratamento superovulatório. Botucatu - SP, 1991.

\begin{tabular}{|c|c|c|c|c|c|}
\hline \multirow[b]{2}{*}{$\mathrm{ANO}$} & \multirow[b]{2}{*}{$\begin{array}{l}\text { NĹMEIRO } \\
\text { IDF } \\
\text { ANIMAIS }\end{array}$} & \multicolumn{4}{|c|}{ REACAOOOVARIANA } \\
\hline & & $\begin{array}{l}\text { OVARIO } \\
\text { CONA } \\
\text { CORPOS } \\
\text { LITTEOS }\end{array}$ & $\begin{array}{l}\text { OVÁRIOSCON } \\
\text { HOLICCLOS } \\
\text { ANOVIIIATORIO }\end{array}$ & $\begin{array}{l}\text { (NRRIO) } \\
\text { NAO } \\
\text { REATIVO }\end{array}$ & $\begin{array}{l}\text { OVARIO } \\
\text { ADI:RII) }\end{array}$ \\
\hline 1989 & 21 & 14 & 7 & 0 & 0 \\
\hline 1990 & 22 & 11 & $\underline{2}$ & 2 & 7 \\
\hline TOTAL & 43 & 25 & 9 & 2 & 7 \\
\hline
\end{tabular}

\section{DISCUSSÃ()}

Veste experimento trabalhou-se com um grupo de caprinos. portadores de translocaç̧ão cromossômica 5/15. Lespécie que revelou ser bastante sensível alos tratamentos hormonais indutores do estro e múltiplas ovulações. Neste aspecto. concordam plenamente NISHIKAWA c ONUMA" (1963). 
PRLSTES, N.C.; MEIRA. ( ; BONFIM, C.A.M.: BICUDO, S.D.; LOPES. M.I).: PAPA. F.O. Estudo da técnica de colela, congelação e descongelaçāo de embriōes de cuprinos (Capra hircus). da raça Saancn, portadores da translocação 5/15. Braz. .J. vet. Res. anim. Sci., São Paulo, v.30, p.249-54, 1993. Suplemento.

Os primeiros passos foram dados por $\triangle$ MOROSO el al.' (1942) que promoviam a detecção visual do cio normal, pois não se conheciam técnicas indutoras e as drogas cram escassas e de pureza duvidosa.

Foram empregados programas bascados no uso da esponja intravaginal impregnada com progesterona, aplicação de Cloprostenol e ISSH-P em injeções intramusculares como métodos indutores do estro e superovulação. Para a deteç̧ão do cio foi utilizado rufião vasectomizado. Mctodologia semethante foi descrita por MOORE? (1974).

Dos 43 animais submetidos aos programas, em 3 não foi observado cio, 18 exteriorizaram sinais em 24 horas, 1 em 36 horas e $21 \mathrm{~cm} 48$ horas após a remoção da esponja (Tab. 1). Resultados concordantes foram obtidos por NUTI et al. ${ }^{10}$ (1987). Foi observada a presença de corpos lúteos nos ovários, sugerindo resposta ao tratamento superovulatório imposto (Tab. 4).

Os meios de coleta sofreram, no decorrer do tempo, inúmeras modificações, evoluindo para substâncias puras e quimicamente conhecidas.

Procedeu-se a técnica de coleta cirúrgica, via flanco, que permitiu total exposição do trato reprodutivo, propiciando fácil lavagem das tubas e úlero. $A$ laparotomia foi efetuada 5 a 6 dias após a cobertura, utilizando-se PBS a $37^{\circ} \mathrm{C}$ como meio de coleta. Técnicas semelhantes foram descritas por MOORE? (1974): NLTI et al. "1 (1987). Foram recuperadas 71 estruturas de 43 animais, devendo-se ressaltar que alguns animais redundaram em colcta negativa, embora apresentassem excelente reação ovariana (Tab. 2). Impressões e resultados semelhantes foram descritos por NUTI et al. ${ }^{10}$ (1987). Estc fato pode ser atribuído aos repetidos tratamentos hormonais, às falhas na técnica de coleta, ou ser devido a provável subfertilidade dos animais translocados.

A formação de aderências decorrentes do manuseio, representou o ponto crítico e por vezes limitante das repetidas coletas.

Em pequenos ruminantes, particularmente caprinos, pouco tem sido estudado a respeito da biotecnologia embrionária. É pouco seguro extrapolar resultados de congelação de embriões de uma espécic para outra, pois há sensíveis diferenças quanto à fase de desenvolvimento, curva de congelação e grau de tolerância aos agentes crioprotetores, no que concordam POLGE c WILLADSEN"1 (1978).

A descongelação de 38 embriōes preservados em Glicerol redundou em fracasso de desenvolvimento em cultivo. Em todos os cmbriões cultivados imediatamente após a coleta ou criopreservados com o 1-2 Propanodiol, foi constatado $100 \%$ de viabilidade c crescimento no meio de cultura (Tab. 3). Resultados semelhantes foram relatados por BILTON; MOORE (1976); BOUTRON ${ }^{4}$ ( 1984 ) c HALASZ; COLLINS $^{\dagger}$ (1984); WILIT et al. ${ }^{13}$ (1986); FRIELDLER et al. ${ }^{5}$ (1988).

O envasamento c congelação seguiram padrões clássicos da curva, resfriamento, cristalizaçāo, congelação, armaze-namento, com o cilindro de aço adaptado na boca do botijão de Nitrogênio líquido. A descongelação foi promovida, mergulhando as palhetas em água a $37^{\circ} \mathrm{C}$ por 20 segundos, semelhante ao proposto por BARIL et al. ${ }^{2}$ (1989).

A descongelação e reidratação foram executadas levando os embriões a três passagens sucessivas contendo sucrose que. pelo fato de não penetrar na célula, teve efeito osmótico significalivo, reduzindo o dano celular. Esta técnica foi coincidente à apresentada por FRIELDLER et al. ${ }^{5}$ (1988) e BARIL et al. ${ }^{2}$ (1989).

\section{CONCLUSÕES}

- A maioria dos animais reagiu bem ao tratamento indutor de cio e método superovulatório;

- Em todos os animais tratados com esponja intravaginal, observou-se uma vaginitc após sua remoção;

- A laparotomia via flanco permite fácil acesso e lavagem do útero e tubas para coleta de embriões;

- A média de embrióes oblida foi considerada satisfatória para o grupo de animais estudados;

- Foram obscrvadas aderências dos genitais após repetidas laparotomias;

- O 1-2 Propanodiol mostrou-se mais efetivo crioprotetor que o Glicerol;

- Os embriōes congelados com o Glicerol não se desenvolveram em cultivo, ao contrário daqueles cultivados à fresco ou preservados com 1-2 Propanodiol. 
PRESTES, N.C.: MI:IRA, C.; BONIIM. C.A.M.; BICUDO. S.D.: LOPLS. M.D.: PAPA, F.O. F.studo da lécnica de coleta. congelação e descongelação de embriōes de caprinos (Capra hircus). da raça Saanen, portadores da translocaçăo 5/15. Braz. J. vet. Res. anim. Sci.. São Paulo, v.30, p.249-54, 1993. Suplemen(o).

\title{
SUMMARY
}

\begin{abstract}
This work studied the surgical technique for collecting embryos in the goat. using heterozygous donors for the $5 / 15$ translocation, which were bred with translocated animals. At the same, time technical characteristics related to cryopreservation, thawing and cultivation of the structures were performed. Considering the superovulatory treatment, oestrus was observeci 24 or 48 hours after removing the intravaginal sponge and 71 viable structures were yielded in 43 collections. The 1-2 Propanediol proved to be an efficient cryoprotectant agent. and allowed the cultivation of all the embryos. This result was not observed when glycerol was used as a cryoprotectint agent. Adhesions due to multiple collections constitute a great challenge for the improvement of this technique. since they are considered a limiting factor to the use of donors in the beginning of their reproductive lives.
\end{abstract}

UNITERMS: Embryos; Cryopreservation: Thawing: Goats

\section{REFERENCIAS BIBLIOGRÁFICAS}

01-AMOROSO, E.C.; GRIFFITHS, W.F.B.; HAMILTON, W.G. The early development of the goat (Capra hircus). Vet. Rec., v.51, p.377-404, 1942.

02-BARIL, B.; CASAMITJANA, P.; PERRIN, J.; VALLET, J.C. Embryo production, freezing and transfer in Angora, Alpine and Saanen goats. Zuchthyggiene. v.24, p.101-11, 1989.

03-BILTON, R.J.; MOORE, N.W. In vitro culture, storage and transfer of goat embryos. Aust. J. Biol. Scio, v.29, p. 125-9, 1976.

04-BOUTRON, P. More accurate determination of the quantity of ice crystallized at low cooling rates in the glycerol and 1,2-Propanediol aqueous solutions: Com-

05-FRIELDLER, S.; GIUDICE, L.C.; LAMB, E.J. Cryopreservations of embryos and ova. Fertil. and Steril., v. 49, n. 5, p. $743-64,1988$.

(16-HALASZ, N.A.; COLLINS, G.M. Studies in cryopreservation II Propylene glycol and glycerol. Cryobiology, v.21, p. 144-7, 1984.

07-MOORE, N.W. Multiple ovulations and ovum transfer in the goat. Proc. Aust. Soc. Anim. Prod., v. 10, p.2469, 1974.

08-MOORE, N.W. Techniques and advances in the recovery storage and transfer of embryos in the goat. In: INTERNATIONAL CONFERENCE OF GOATS, 4., Brasilia, 1987. Proceedings. Brasília- EMBRAPA, 1987. v.1, p.587-99.
09-NISHIKAWA, Y.; ONUMA, H. Studies on the transplantation of ova (artificial pregnancy) in goats. Proc. Jap. Acad., v.39. p.519-24, 1963.

10-NUTI, L.C.; MINHAS, B.S.; BAKER, W.C.;CAPEHART, J.S.; MARRACI, P. Superovulation and recovery of zygotes from Nubian and Alpine dairy goats. Theriogenology, v.28, n.4, p.481-9, 1987.

11-POLGE, C.; WILLADSEN, S.M. Freezing eggs and embryos of farm animals. Cryobiology, v.15, p.3703,1978 .

12-SOUZA, R.B.: DE BEM, A.R. Congelamento de embriões isocriogen. In: REUNIÃO ANUAL E I REUNIÃO INTERNACIONAL DA SOCIEDADE BRASILEIRA DE TRANSFERÊNCIA DE EMBRIÕES. 3., Santa Maria, 1988.

13-WILDT, D.E.; SCHIEW, M.C.; SCHMIDT, P.M.: GOODROWE, K.L.; HOWARD, J.G.; PHILLIPS, L.G.; O'BRIEN, S.J.; BUSH, M. Developing animal model systems for embryo technologis in rare and andangered wildlife. Theriogenology, v.25, n. 1, p.3349, 1986. 